\title{
Continuity of care in general practice is associated with fewer ambulatory care sensitive hospital admissions: a cross-sectional study of routinely collected, person-level data
}

\author{
Authors: Isaac Barker, Adam Steventon and Sarah Deeny
}

\begin{abstract}
Aims
Continuity of care with a GP is valued by physicians and patients as a key aspect of high-quality care. We assessed whether continuity is associated with hospital admissions for ambulatory care sensitive conditions, which are those considered manageable in primary care.
\end{abstract}

\section{Methods} patients aged between 62 and 82 years $(n=230,472)$ from 200 general practices participating in the Clinical Practice Research Datalink in England. We measured continuity of care using the Usual Provider of Care (UPC), which is defined as the proportion of contacts with the most regularly seen GP during the period from April 2011 to March 2013.

We performed regression analysis to test the association between UPC and the number of ambulatory care sensitive admissions to hospital per person between April 2011 and March 2013. We controlled for demographic and clinical patient characteristics and allowed for the clustering of individuals within general practices. We performed subgroup analysis to examine whether the relationship between continuity of care and admissions differed according to how often patients used general practice and their age.

\section{Results}

Across all patients, the average UPC was 0.61 , and this was lower among practices with more GPs (large practices average UPC 0.59, small practices average UPC 0.70). Higher continuity of care was associated with fewer admissions for ambulatory care sensitive conditions. When modelled, controlling for other factors, an increase of UPC by 0.2 for all patients would reduce these admissions by $6.3 \%$ (95\% CI, 7.68-4.97\%). We found no evidence of heterogeneity in the relationship between continuity of care and ambulatory care sensitive conditions
We analysed linked primary and secondary care records for

admissions between age groups, but there was greatest evidence of an association among heavy users of general practice care (who also experienced more of these admissions: 0.34 in the 5th quintile vs 0.04 in the 1 st quintile).

\section{Conclusions}

Strategies that improve continuity of care may reduce avoidable hospital admissions, particularly among the highest users of healthcare. Previous studies have found that continuity of care is associated with patient satisfaction, and it aligns closely with the motivations of those working in general practice. Therefore, quality improvement strategies that target increased continuity have the potential to improve patient and professional experience as well as cost, although specific interventions would need to be evaluated. This study shows that continuity of care can be measured at person-level using data from the electronic medical record, which are readily available to general practice teams for use in quality improvement.

\section{Conflict of interest statement}

NA.

Authors: The Health Foundation, London, UK 